\title{
Annular illumination photoacoustic probe for needle guidance in medical interventions
}

Rascevska, Elina, Kalloor Joseph, Francis, Manohar, Srirang

Elina Rascevska, Francis Kalloor Joseph, Srirang Manohar, "Annular illumination photoacoustic probe for needle guidance in medical interventions," Proc. SPIE 11077, Opto-Acoustic Methods and Applications in Biophotonics IV, 110770L (19 July 2019); doi: 10.1117/12.2527080 


\title{
Annular illumination photoacoustic probe for needle guidance in medical interventions
}

\author{
Elina Rascevska ${ }^{\mathrm{a}}$, Kalloor Joseph Francis ${ }^{\mathrm{a}}$, and Srirang Manohar ${ }^{\mathrm{a}}$ \\ ${ }^{a}$ University of Twente, Multi-Modality Medical Imaging, Technical Medical Centre, Enschede, \\ The Netherlands
}

\begin{abstract}
During percutaneous medical interventions, accurate needle placement and advancing to the target of interest is required to avoid complications and to improve clinical outcomes. Therefore, we present a novel photoacoustic (PA) imaging-based approach as a complement to conventional ultrasound (US) imaging for visualization and guidance of interventional needles. To overcome the limitations associated with light penetration with the conventional extracorporeal illumination, we propose an interstitial light delivery to the target of interest by use of a custom-made annular illumination probe (AIP). This probe accommodates an interventional needle (14 gauge) within its lumen, allowing to advance both tools and acquire real-time PAUS images simultaneously. The light is delivered utilizing 72 multimode optical fibers arranged around the circumference of the hollow center of the AIP. Preliminary results show that PA images obtained with the AIP provide with good complementary contrast to the US imaging for visualization of the interventional needles and its guidance to an absorbing target within chicken breast tissue.
\end{abstract}

Keywords: Photoacoustic, Ultrasound, Interstitial illumination, Imaging, Radiofrequency Ablation, Biopsy, Surgical tool tracking, Needle guidance

\section{INTRODUCTION}

In interventional procedures, US imaging is commonly used for percutaneous needle advancing to a target of interest that is typically located several centimeters beneath the skin surface. Accurate needle guidance is required for treatment efficiency and to avoid complications such as a neural or vascular puncture. ${ }^{1}$ However, needle visualization in an US image can be challenging due to specular US reflections from the angled needle not intersecting the transducers aperture and multiple artifacts, such as reverberations, shadowing, comet tail. ${ }^{2}$

PA imaging is known to be an excellent complement to US imaging for absorbing target visualization, such as blood vessels and metallic objects. Absorption of metal is a few orders in magnitude higher than that of tissue, therefore PA imaging provides good contrast between the needle and the surrounding tissue. ${ }^{3,4}$ Moreover, relatively high blood absorption and hyper-vascularisation of tumors have shown to offer good contrast in PA imaging. ${ }^{5,6}$ Most PA systems proposed so far use extracorporeal illumination approach where the light illumination and acoustic detection is done at the surface of the skin. A fundamental constraint of extracorporeal PA imaging is the limited imaging depth in absorbing tissue. Several groups have tried to tackle this limitation with interstitial tissue illumination. ${ }^{3,7,8}$ In this approach light is directly delivered to the region of interest and the photoacoustic signal can be detected at the surface with conventional US transducer.

We propose a novel interstitial light delivery method to enable deep tissue imaging for needle guidance in interventional procedures by use of AIP. This probe has a hollow center allowing to accommodate the interventional needle within it, and 72 multimode optical fibers arranged around its circumference for light delivery. This allows for real-time PA imaging while simultaneously advancing the needle and the annular illumination probe to the target. In this article, we describe the design of the probe and its application in radio-frequency ablation and biopsy needle guidance.

Further author information: Send correspondence to Kalloor Joseph Francis

E-mail: f.kalloorjoseph@utwente.nl, Telephone: +31 616975582

Opto-Acoustic Methods and Applications in Biophotonics IV, edited by Vasilis Ntziachristos,

Roger Zemp, Proc. of SPIE-OSA Vol. 11077, 110770L · C) 2019 SPIE-OSA

CCC code: $1605-7422 / 19 / \$ 21 \cdot$ doi: $10.1117 / 12.2527080$

Proc. of SPIE-OSA Vol. 11077 110770L-1 


\section{MATERIALS AND METHODS}

The annular illumination PA probe (Fig.1, right) consists of 2 steel ferrules, one with an inner diameter of 2.8 $\mathrm{mm}$ and the outer diameter of $3.2 \mathrm{~mm} .72$ multi-mode optical fibers $(N A=0.2,100 \mu \mathrm{m})$ are arranged in annular manner around the circumference of the inner ferrule (Fig.1, right B). The hollow center of the ferrule allows accommodating the 14-gauge interventional needle. At the distal end the fibers are bundled together (Fig.1, right C) In our system (Fig.1, left) optical excitation for PA imaging is provided by a Quanta-Ray Lab 170 (Spectra Physics) Nd:YAG laser connected to a VersaScan-L 532/BB/HE/800 optical parametric oscillator (OPO). For these experiments, OPO is set to a wavelength of $680 \mathrm{~nm}$ by software GWU ScanMaster. The corresponding pulse length is in range of 5-10 ns. The laser is coupled to the fiber bundle using a microlens array and a planoconvex lens. For imaging Alpinion Ecube 12R (Seoul, Korea) ultrasound machine with linear probe L3-12 (center frequency $8.5 \mathrm{MHz}$ ) is used. Acquisition sequence consists of laser triggering Alpinion $(10 \mathrm{~Hz})$ and combined PA and US imaging.
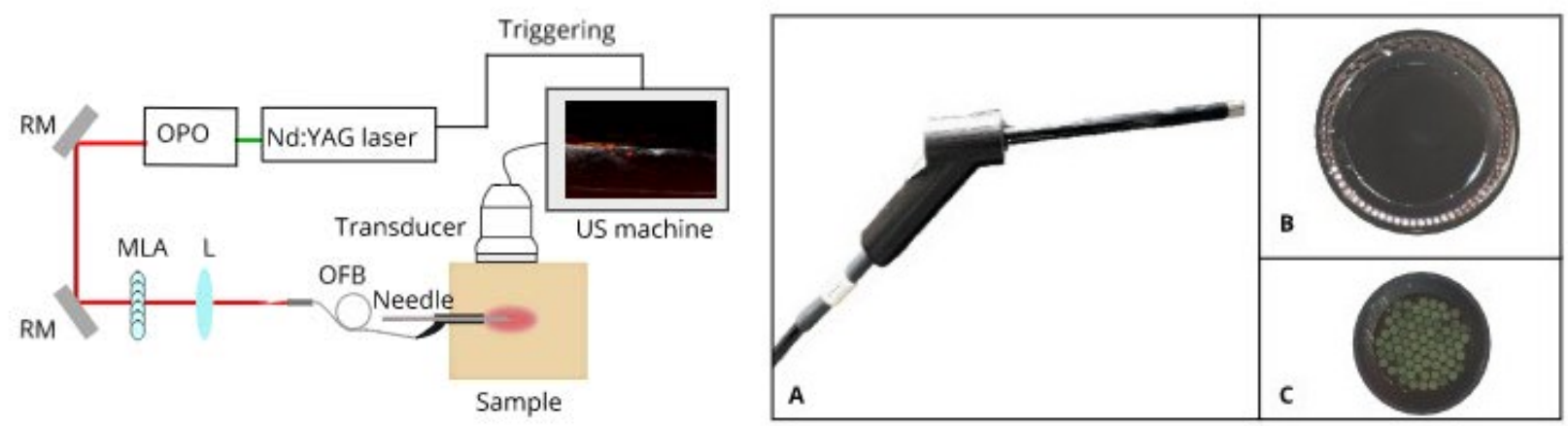

Figure 1: (Left) PA imaging setup. OPO - optical parametric oscillator, RM - reflecting mirror, MLA - microlens array, L- plano-convex lens, OFB - optical fiber bundle. (Right) A - Annular illumination PA probe, B - proximal end of the probe, $\mathrm{C}$ - distal end of the probe.

A radio-frequency ablation (RFA) needle was slid into the lumen of the annular PA probe and inserted into an imaging volume consisting of layered chicken breast tissue (Fig.2a). Expandable RFA electrodes (tines) were deployed up to a point where the visibility of them in PA image was lost. PA and US images were reconstructed using conventional sum and delay beamformer and averaged over 20 frames.

To demonstrate guidance of a biopsy needle, an absorbing target was developed using a piece of chicken breast tissue dabbed with India ink and positioned in-between 2 layers of chicken breast tissue (Fig.2e). The biopsy needle was inserted into the lumen of the annular probe and advanced to this target guided by PA imaging.

\section{RESULTS}

In this section, we present our results from needle guidance experiments for two potential applications namely, radio-frequency ablation and biopsy.

The schematic in figure 2a shows the AIP encompassing the RFA needle with multiple electrodes (tines) deployed into the tissue. Figure $2 \mathrm{~b} \& \mathrm{c}$ are PA and US images respectively. Combined PA and US image is shown in figure 2d. PA image provides a higher contrast compared to the US for the RFA needle and the tines. Contrast to noise ratio of PA is $8.2 \pm 0.3$ (standard deviation) while for the US it is $1.1 \pm 0.2$. The combined PA and US image in figure $2 \mathrm{~d}$ provides both structural information of the tissue and the high contrast of the needle.

The schematic in figure 2e shows guidance of the biopsy needle along with AIP to a tumor mimicking tissue. Both the needle and the target tissue are clearly visible in the PA image in $2 \mathrm{f}$. While the US image in $2 \mathrm{~g}$ shows a shadow artifact from the metal needle marking its location with a small intensity difference at the boundary of the target tissue. Combined PA and US image is shown 2h. The contrast of the needle in PA is $5.4 \pm 2.9$ and 


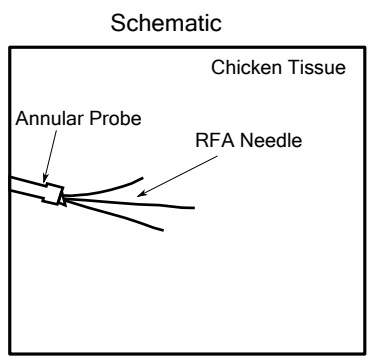

(a)

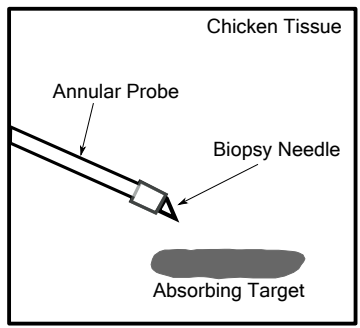

(e)

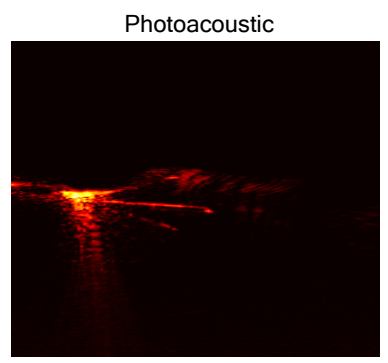

(b)

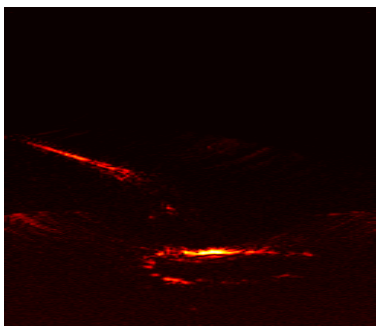

(f)

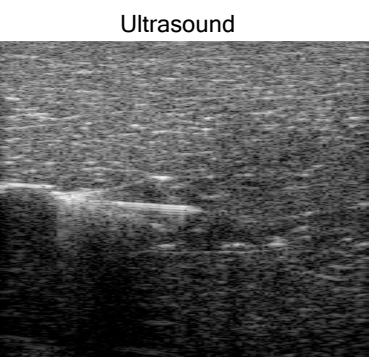

(c)

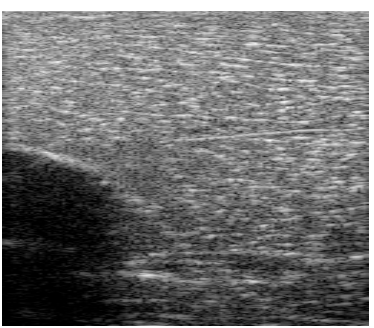

(g)

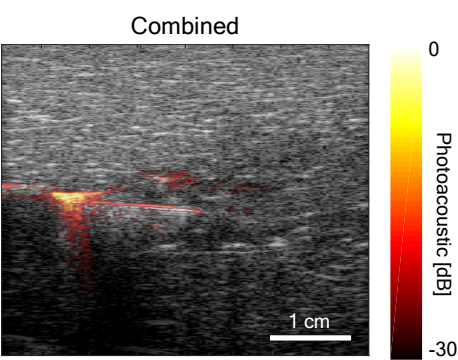

(d)

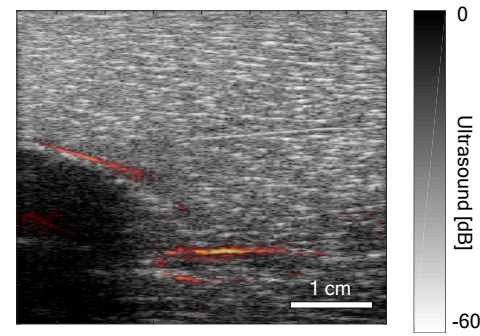

(h)

Figure 2: (a) Schematic of the imaging plane with AIP and RFA needle, (b) PA image of the RFA needle, (c) US image of the RFA needle, (d) overlaid PA and US image of the RFA needle, (e) schematic of the imaging plane with AIP and biopsy needle, (f) PA image of the absorbing target and biopsy needle, (g) US image of the absorbing target and biopsy needle, (h) overlaid PA and US image of the absorbing target and biopsy needle.

in the US it is $0.8 \pm 0.3$. The contrast of the target tissue in the PA image is $11.8 \pm 2.0$, while in the US image it is $0.4 \pm 0.1$.

In both the experiments with needle guidance of RFA and biopsy needle, the contrast of needle in the PA image is better compared to the US. Additionally, as most tumors have high vascularization resulting in an increased optical absorption, PA imaging can help needle guidance to the target. ${ }^{7}$ Hence, the proposed interstitial PA imaging method can be used in device guidance in interventional procedures.

\section{CONCLUSIONS}

We have proposed and developed an interstitial annular illumination probe for PA imaging with potential applications in needle guidance in medical interventions to improve needle placement accuracy and potentially procedure quality. Preliminary results have shown that annular PA probe can be used as a complement to conventional ultrasound imaging to (i) visualize interventional needles and (ii) guide needles to absorbing targets. Future work will investigate the utility of the system in imaging the effects and extent of the ablation procedure.

\section{REFERENCES}

[1] Chin, K. J., "Needle and Transducer Manipulation : The Art of Ultrasound-guided Regional Anaesthesia," 1(April), 27-32 (2010).

[2] Reusz, G., Sarkany, P., Gal, J., and Csomos, A., "Needle-related ultrasound artifacts and their importance in anaesthetic practice," 112(February), 794-802 (2014).

[3] Bell, M. A. L., Kuo, N. P., Song, D. Y., Kang, J. U., and Boctor, E. M., "In vivo visualization of prostate brachytherapy seeds with photoacoustic imaging with photoacoustic imaging,"

[4] Su, J. L., Karpiouk, A. B., Wang, B., and Emelianov, S. Y., "Photoacoustic imaging of clinical metal needles in tissue," Journal of biomedical optics 15(2), 021309 (2010).

[5] Francis, K. J., Chinni, B., Channappayya, S. S., Pachamuthu, R., Dogra, V. S., and Rao, N., "Characterization of lens based photoacoustic imaging system," Photoacoustics 8, 37-47 (2017). 
[6] Francis, K. J., Chinni, B., Channappayya, S. S., Pachamuthu, R., Dogra, V. S., and Rao, N., "Two-sided residual refocusing for an acoustic lens-based photoacoustic imaging system," Physics in Medicine $G_{3}$ Biology 63(13), 13NT03 (2018).

[7] Piras, D. and Schütte, P., "Photoacoustic needle : minimally invasive guidance to biopsy Photoacoustic needle : guidance to biopsy," (2018).

[8] Rebling, J., Oyaga Landa, F. J., Deán-Ben, X. L., Douplik, A., and Razansky, D., "Integrated catheter for simultaneous radio frequency ablation and optoacoustic monitoring of lesion progression," Optics Letters 43, 1886 (apr 2018). 